\title{
La naissance, un moment particulier à haut risque
}

\section{Birth, a particular high-risk moment}

\author{
C. Boithias $\cdot$ J.-F. Tolsa \\ (C) Lavoisier SAS 2018
}

La naissance représente un début, le début d'une vie conditionnée par les circonstances de l'accouchement et des premières minutes de vie.

Un événement naturel, physiologique à accompagner avec la plus grande bienveillance et expérience. Environ $10 \%$ des naissances requièrent une intervention professionnelle pour la prise en charge de nouveau-nés en détresse, d'un trouble de l'adaptation transitoire à une réanimation instrumentalisée et médicamenteuse nécessitant la mise en place rapide de soins intensifs spécialisés dans $1 \%$ des situations.

Une naissance n'est pas seulement le début d'une vie, mais aussi la fin d'un long voyage, celui de l'histoire d'une conception, des conditions rencontrées pendant la grossesse et des circonstances de l'accouchement. La connaissance de tout ce chemin d'histoire est capitale pour une prise en charge adéquate d'un nouveau-né.

Les circonstances amenant sages-femmes, obstétriciens et pédiatres-néonatologistes à évaluer, à intervenir, sont multi- ples, parfois imprévisibles et requièrent souvent des actions précises, rapides et coordonnées.

Cette revue propose d'aborder des situations fréquemment rencontrées en salle de naissance, incluant les pratiques fondées sur l'évidence, le monitorage, le dépistage de cardiopathies congénitales à risque critique non diagnostiquées en anténatal, des recommandations de bonnes pratiques en cas de liquide méconial, de la prise en charge de la douleur des nouveau-nés et des modalités de transport en urgence vers une unité de réanimation dans différents centres européens.

Ces mises au point nous confrontent et nous guident dans nos évaluations et prises en charge délicates à la naissance, des actions nécessitant régulièrement des remises en question et des adaptations de nos pratiques.

La naissance, ce moment si particulier qui conditionne le début de la vie et le reste de la vie, un moment qui est parfois suspendu dans nos mains.
C. Boithias $(\bowtie)$

Service de réanimation pédiatrique et médecine néonatale, hôpital de Bicêtre, 78, rue du Général-Leclerc,

F-94270 Le Kremlin-Bicêtre, France

e-mail : claire.boithias@aphp.fr

J.-F. Tolsa $(\bowtie)$

CHUV Département femme-mère-enfant

Rue du Bugnon 21 CH-1011 Lausanne, Suisse

e-mail : jean-francois.tolsa@chuv.ch 